\title{
Review: existing evidence does not support a survival benefit for women with metastatic breast cancer who participate in group psychological interventions compared with usual care
}

Edwards AG, Hailey S, Maxwell M. Psychological interventions for women with metastatic breast cancer. Cochrane Database Syst $\operatorname{Rev} 2004 ;(2): C D 004253$.

Do psychological interventions (education, individual cognitive behavioural or psychotherapeutic programmes, or group support) improve survival and psychological outcomes in women with metastatic breast cancer?

\section{METHODS}

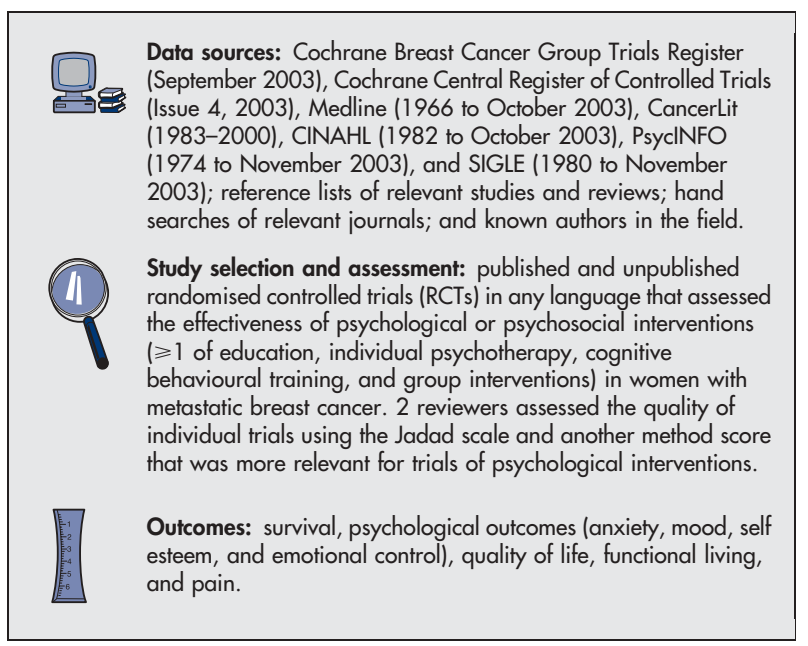

\section{MAIN RESULTS}

5 studies $(\mathrm{n}=636)$ met the selection criteria. 2 studies assessed cognitive behavioural group interventions, and 3 assessed supportiveexpressive group therapy. Meta-analysis using a fixed effects model showed that group psychological interventions did not differ from usual care for survival at 1,5 , or 10 years (table). Cognitive behavioural therapy did not differ from usual care for anxiety at 6 months ( 1 trial); Profile of Mood States at 6 months ( 1 trial) or 8 months ( 1 trial); self esteem at 6 months ( 1 trial); or Functional Living Index at 8 months (1 trial). Supportive-expressive group therapy improved scores on the Courtauld Emotional Control scale at 8 months ( 1 trial) and reduced reported pain assessed using a 10 point visual analogue scale (meta-analysis of 2 trials, weighted mean Swansea Clinical School, University of Wales Swansea, Swansea, Wales, UK. adriangkedwards@btinternet.com

Source of funding: Cochrane Health Promotion and Public Health Field (Australia) difference 0.75 reduction, $95 \%$ CI 0.63 to 0.86 ) compared with usual care; the groups did not differ for Profile of Mood States at 10-12 months ( 2 trials) or quality of life at 1 year ( 1 trial)

\section{CONCLUSIONS}

Existing evidence does not support a survival benefit for women with metastatic breast cancer who participate in group psychological interventions (cognitive behavioural therapy or supportive-expressive therapy) compared with those who receive usual care. Evidence on the effects on various aspects of psychological functioning is mixed.

\section{Commentary}

- dwards et al provide a rigorous review of RCTs comparing the - effectiveness of psychological interventions on biopsychosocial outcomes in women with metastatic breast cancer. Only 5 eligible RCTs were identified, and these had diverse psychological outcome measures and conflicting results. Because of the diverse nature of the studies and participants, the authors used a rigorous approach to data analysis that considered the heterogeneity and scarcity of data in the field as potential factors that could affect the results of the meta-analysis. The authors also considered potential effect modifiers such as family history of breast cancer, age, time of diagnosis of metastasis, variation in length of follow up, and loss to follow up.

The results are relevant for oncology nurses, psychiatric clinical nurse specialists, and nurse practitioners working in hospital or community oncology settings, as well as managers responsible for development and implementation of programmes for women with metastatic breast cancer. Evidence from this review highlights the equivocal nature of existing data on the effectiveness of group psychological interventions for women with advanced breast cancer. Although the evidence suggests some short term psychosocial benefits, these benefits were not maintained in the longer term. These findings are disappointing considering the conceptually logical and anecdotal evidence of the benefits of such psychosocial interventions. Considering the human and financial resource commitment of breast cancer services to psychosocial interventions, the lack of evidence supporting the efficacy of this approach justifies evaluation of traditional programming and resource allocation for this patient population. Moreover, the design of future studies should consider the authors' excellent methodological critique of the extant literature.

Judith Haber, RN, PhD, APRN, BC, FAAN Division of Nursing, New York University New York, New York, USA

Group psychological interventions $v$ usual care for women with metastatic breast cancer*

\begin{tabular}{lllll}
\hline & & \multicolumn{2}{c}{ Weighted event rates } & \multirow{2}{*}{ Outcomes } \\
\cline { 3 - 4 } & Number of trials $(\mathbf{n})$ & Psychological intervention & Usual care & RBI or RBR (95\% Cl) $\dagger$ \\
\hline Survival at 1 year & $4(511)$ & $72 \%$ & $70 \%$ & RBI 3 (-8 to 15) \\
Survival at 5 years & $4(511)$ & $22 \%$ & $25 \%$ & RBR 13(-19 to 37) \\
Survival at 10 years & $3(276)$ & $11 \%$ & $13 \%$ & RBR 19\% (-54 to 58) \\
\hline
\end{tabular}

*Abbreviations defined in glossary; weighted event rates, RBI, RBR, and Cl calculated from data in article based on a fixed effects model. †Not significant. 